\section{Microbiome-based companion diagnostics: no longer science fiction?}

\author{
J Raes ${ }^{1,2}$
}

In the last decade, our knowledge on the role of the gut microbiome in health and disease has greatly increased, accompanied by an unseen hype around both its diagnostic and therapeutic potential. Yet, one application area of the microbiome has thus far remained understudied: its role as guidance for therapeutic decisions, treatment monitoring and prediction of response.

In this issue of Gut, Mondot et $a l^{1}$ investigate to what extent the microbiome can both inform us on as well as contribute to treatment outcome in resection surgery for Crohn's disease (CD). Although often the only resort next to dilatation, ${ }^{2}$ this intervention is not curative, with first-year endoscopic recurrence rates at $28-93 \%$ and frequent necessity of reoperation. ${ }^{3}$ As such, there is an important need for effective postoperative maintenance strategies and predictions for postoperative outcome.

In a longitudinal study of both the faecal and mucosa-adherent microbiota in 20 patients undergoing resection surgery, Mondot et al describe the microbial groups recolonising the intestinal lining around the anastomosis and show different trajectories in recurring patients versus those in remission; the latter were found to exhibit a stable, cohesive community structure, which is spatially homogeneous before and after the anastomosis. The species associated with recurrence could form companion drug targets for this procedure, although their causal role still needs to be established. A particularly intriguing aspect of this study is the identification of multiple operational taxonomic units whose presence before surgery seems predictive of early recurrence. Signatures like these open up the exciting prospect of a quick companion diagnostic that would allow a preoperative evaluation whether the patient is likely to remain in long-term remission after treatment. Of course, the current study is

${ }^{1}$ Department of Microbiology and Immunology, Rega Institute, KU Leuven-Leuven University, Leuven, Belgium; ${ }^{2}$ VIB Center for the Biology of Disease, Leuven, Belgium

Correspondence to Dr J Raes, Department of Leuven-Leuven University, Herestraat 49, Leuven B-3000, Belgium; jeroen.raes@med.kuleuven.be Microbiology and Immunology, Rega Institute, KU underpowered; larger scale validation in multiple centres will be needed before such a companion diagnostic becomes reality, but conceptually this is a very attractive model.

Thus far, similar studies are scarce, but equally promising. For example, Rajca et $a l^{4}$ showed that low rates of Faecalibacterium prausnitzii and Bacteroides are predictors of relapse after infliximab withdrawal in CD. Machiels et $a l^{5}$ found the presence of Ruminococcus gnavus, Bacteroides vulgatus and Clostridium perfringens and the absence of Blautia and Roseburia in faecal samples of patients with UC before surgery to be associated with a higher risk of pouchitis after ileal pouch-anal anastomosis. Kaddurah-Daouk et $a l^{6}$ identified two secondary, bacterial-derived bile acids in a metabolomics screen that contributed to predicting the magnitude of statin-induced low-density lipoprotein cholesterol lowering in responders. Vétizou et $a l^{7}$ show that the success of ipilimumab anticancer treatment is even causally dependent on the presence of Bacteroidales. Together, these studies suggest that the role of the microbiome in clinical guidance is relevant in a wide range of conditions and interventions.

Perhaps, the most likely therapeutic area where microbiome-based treatment guidance is that where the microbiota is part of the treatment itself: faecal microbiota therapy (FMT), also known as faecal transfer. FMT has been extremely effective in Clostridium difficile infections, yet for other pathologies success rates are more limited or unclear, and microbiomeinformed treatment steering might be a solution. One such area is that of UC. Studies seem to agree upon a $\pm 25-30 \%$ success rate. ${ }^{8-10}$ Yet, as the placebo efficacy was of a similar order of magnitude in some studies, ${ }^{9}$ the initial enthusiasm was somewhat curbed. To improve upon these numbers, microbiome-based treatment guidance could be an option. As a point in case, we recently found that donor richness and the number of transferred phylotypes were associated with sustained remission and found indications that FMT success was associated with the successful transfer of Roseburia and Oscillibacter. ${ }^{8}$ Although still early days, this does suggest that microbiome-based patient and donor selection, with the latter ultimately replaced by construction of personalised probiotic cocktails, is likely to benefit FMT outcome in UC, and possibly in other pathologies.

Overall, these studies indicate that the microbiome field is slowly but surely approaching the clinic. I believe the future role of microbiome monitoring in daily medical practice can be found at four different levels (figure 1). First, microbiome markers can be used for diagnosis (and potentially prognosis) of disease. Second, analysis of patient microbiota could predict the outcome of treatment options. Third, based on the patient's microbiome, a personalised treatment strategy can be devised, be it based on the administrations of specific microbial cocktails ('precision probiotics'), targeted microbial nutrients ('precision prebiotics'), personalised dietary interventions or targeted antibiotics and phages. Finally, treatment success and establishment of normobiosis can be monitored using microbiome timeseries analysis. ${ }^{11}$ The thrilling fact that multiple aspects of this microbiome-based therapeutic model are nearing clinical implementation reflects how the field is shaking off its 'growing pains' and is increasingly becoming a true translational discipline.

Acknowledgements I thank Marie Joossens for valuable input on this manuscript. The Raes Lab is supported by the Research Foundation Flanders (FWO),
Figure 1 Microbiome-based therapeutic model. 
the Flemish Agency for Innovation by Science and Technology (IWT), KU Leuven, FP7 METACARDIS HEALTH-F4-2012-305312 and the Rega Institute.

Correction notice This article has been corrected since it published online first. An Open Access licence has now been added.

Funding Seventh Framework Programme.

Competing interests None declared.

Provenance and peer review Commissioned; internally peer reviewed.

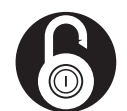

\section{OPEN ACCESS}

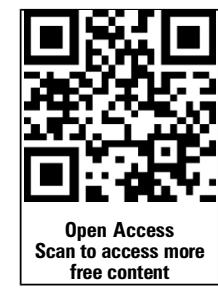

Open Access This is an Open Access article distributed in accordance with the Creative Commons Attribution Non Commercial (CC BY-NC 4.0) license, which permits others to distribute, remix, adapt, build upon this work non-commercially, and license their derivative works on different terms, provided the original work is properly cited and the use is non-commercial. See: http:// creativecommons.org/licenses/by-nc/4.0/
3 Achkar JP, Hanauer SB. Medical therapy to reduce postoperative Crohn's disease recurrence. Am J Gastroenterol 2000;95:1139-46.

4 Rajca S, Grondin V, Louis E, et al. Alterations in the intestinal microbiome (dysbiosis) as a predictor of relapse after infliximab withdrawal in Crohn's disease. Inflamm Bowel Dis 2014;20:978-86.

To cite Raes J. Gut 2016;65:896-897.

Received 14 December 2015

Accepted 28 December 2015

Published Online First 22 January 2016

\section{(5) Linked}

http://dx.doi.org/10.1136/gutjnl-2015-309184

Gut 2016:65:896-897.

doi:10.1136/gutjnl-2015-311015

\section{REFERENCES}

1 Mondot S, Lepage P, Seksik P, et al. Structural robustness of the gut mucosal microbiota is associated with Crohn's disease remission after surgery. Gut 2016;65:954-62.

2 Thienpont C, D'Hoore A, Vermeire $S$, et al. Long-term outcome of endoscopic dilatation in patients with Crohn's disease is not affected by disease activity or medical therapy. Gut 2010;59:320-4. 